\title{
Extinction of Conditioned Eyelid Responses Requires the Anterior Lobe of Cerebellar Cortex
}

\author{
Stephen P. Perrett and Michael D. Mauk \\ Department of Neurobiology and Anatomy, University of Texas Medical School, Houston, Texas 77225
}

We test the hypothesis that the cerebellar cortex is required for the extinction of conditioned eyelid responses in rabbits trained using standard Pavlovian delay procedures. Following 10 daily training sessions during which rabbits achieved asymptotic performance, lesions of the ipsilateral hemisphere of the cerebellar cortex were made by aspiration. The target of these lesions was the anterior lobe, as suggested by previous observations that this region is necessary for the learning-dependent timing of conditioned eyelid responses (Perrett et al., 1993). We report that anterior lobe damage, as indicated by disrupted response timing and confirmed by tissue analysis, produces severe deficits in conditioned response extinction. Postlesion responses show no significant decline over ten training sessions, whereas response timing and extinction are unaffected by lesions that do not include the anterior lobe. These conditioned responses that do not extinguish display stimulus specificity, excluding the possibility that they are unlearned responses unmasked by cerebellar cortex lesions. These observations suggest that Pavlovian eyelid conditioning is mediated by synaptic plasticity in at least two sites and the anterior lobe of the cerebellar cortex influences one of these sites during extinction. Based on these and previous data, we propose the hypothesis that eyelid conditioning can involve plasticity in both the cerebellar cortex and interpositus nucleus and that plasticity in the nucleus is controlled by input from Purkinje cell activity in the cortex. This hypothesis is consistent with observations that the cerebellar cortex may not always be required for the expression of conditioned responses, but it is necessary for response timing and for extinction.

[Key words: Pavlovian eyelid conditioning, nictitating membrane, Hebbian plasticity, Purkinje cells, interpositus nucleus, long-term depression]

A variety of evidence indicates that the cerebellum is an important component of the neural pathways engaged by Pavlovian conditioning of eyelid responses (McCormick and Thompson, 1984; Yeo et al., 1985a,b; Thompson, 1986; Yeo, 1991; Yeo and Hardiman, 1992). Although considerable attention has focused

Received May 31, 1994; revised Sept. 6, 1994; accepted Sept. 15, 1994.

We thank M. Neal Waxham, Garrett T. Kenyon, Phillip M. Steele, Kim M. Huber, Erik Plautz, and Keith S. Garcia for helpful comments. This work was supported by Scholar's Awards from the McKnight Endowment for Neuroscience and the National Down Syndrome Society as well as by the NIMH.

Correspondence should be addressed to Michael D. Mauk, Department of Neurobiology and Anatomy, University of Texas Medical School, Box 20708, Houston, TX 77225.

Copyright (C) 1995 Society for Neuroscience $\quad 0270-6474 / 95 / 152074-07 \$ 05.00 / 0$ on the contributions of the cerebellar cortex and cerebellar nuclei to the acquisition and expression of responses, recent evidence suggests that cerebellar involvement is not limited to these basic aspects of conditioning. For example, lesions of the cerebellar cortex that include the anterior lobe disrupt the learned timing of conditioned responses (CRs; Perrett et al., 1993). These data suggest that identifying all aspects of conditioning affected by lesions of various cerebellar regions will aid in obtaining a comprehensive account of the mechanisms that mediate the cerebellum's contribution to eyelid conditioning. In the present study, we have examined the effect of cerebellar cortex lesions on the extinction of conditioned eyelid responses acquired prior to the lesion.

In lesion studies of this type it is important to insure that pathways necessary for the expression of CRs-such as the anterior interpositus nucleus (McCormick and Thompson, 1984; Yeo et al., 1985a) - have not been damaged. For example, although previous studies have implicated lobule HVI of the cerebellar cortex as an important region for the expression of CRs (Yeo et al., 1985b; Yeo and Hardiman, 1992), functional damage to the interpositus nucleus could not be excluded in these experiments. Since animals in the present study are trained prior to the lesion, retention of CRs indicates that pathways necessary for response expression have not been damaged. Similarly, it is important to ensure that critical regions of the cerebellar cortex have not been inadvertently spared in lesion studies. In this case, the inability of discrete lesions of the cerebellar cortex to abolish or affect acquisition, expression, or extinction may simply reflect that damage to other cortical regions is required. This point is reinforced by recent observations (Perrett et al., 1993) that lesions of the cerebellar cortex disrupt the learned timing of CRs only when they involve the anterior lobe, a region not previously implicated in eyelid conditioning. These data suggest that Purkinje cells in the anterior lobe are involved in eyelid conditioning and that disruption of response timing can be used as a behavioral indication of lesions that (1) include damage to critical regions of cerebellar cortex and (2) spare the interpositus nucleus. For these reasons we have paid particular attention to lesions that disrupt conditioned response timing. We find that these lesions prevent extinction of conditioned eyelid responses.

These data suggest that at least two sites of plasticity mediate the acquisition and extinction of conditioned eyelid responses and that one site is controlled or strongly influenced by input from the cerebellar cortex (at least during extinction). We propose the hypothesis that these sites include the cerebellar cortex and the cerebellar interpositus nucleus and that extinction involves generalized plasticity, that is, reversal of plasticity responsible for acquisition of CRs. 


\section{Materials and Methods}

Animals and surgical procedures. Thirty-one male, albino rabbits (Oryctolagus cuniculus) weighing 2-2.5 kg were used. Treatment of the animals and surgical procedures were in accordance with an approved animal welfare protocol. Using sterile procedures and halothane as anesthetic (1-2\% mixed in oxygen) each rabbit was initially fitted with a bolt cemented to the skull, two stainless steel wires implanted subdermally in the dorsolateral aspect of the left eye, and a small suture loop in the left upper eyelid. Subsequent aspiration lesions of the cerehellar cortex were performed under the same conditions. In all cases, intravenous fluids, antibiotics, and analgesics were administered postsurgery as needed and at least 1 week was allowed for recovery.

Conditioning procedures. Details of conditioning procedures, data acquisition, and data analysis are presented elsewhere (Mauk and Ruiz, 1992; Perrell et al., 1993). Briefly, animals were trained will eillier 0.5 or $5 \mathrm{kHz}$ tone conditioned stimuli (CSs) paired with a coterminating unconditioned stimulus (US, periocular shock) at an interstimulus interval of $500 \mathrm{msec}$. The US was a $50 \mathrm{msec}$ train of constant current cathodal pulses $(200 \mathrm{~Hz})$ delivered at an intensity of $0.5-3 \mathrm{~mA}$. Shock intensity was adjusted for each animal to induce a robust eyeblink without significant activation of the surrounding facial musculature. Daily training sessions consisted of 12 blocks of nine trials presented at an intertrial interval of $30 \mathrm{sec}$. Eight paired CS/US trials and one CS-alone trial comprised each block.

Movements of the unrestrained upper eyelid were measured using a micro-torque potentiometer connected to the suture loop by light gauge stainless steel wire. In this way, eyelid position was transduced into voltage signals that were amplified and digitized at $1 \mathrm{kHz}$. Digitized responses (2000 points per trial) were subsequently analyzed using custom software. Because the reflex unconditioned response is generally superimposed on the peak of the CR during paired trials, only the CSalone trials were analyzed. Eyelid responses $>0.3 \mathrm{~mm}$ and with latencies to onset between $40 \mathrm{msec}$ and $1 \mathrm{sec}$ after CS onset were scored as CRs. Trials were excluded from analysis if the onset of a response $>0.3$ $\mathrm{mm}$ occurred in a $200 \mathrm{msec}$ baseline period or in the first $40 \mathrm{msec}$ after CS onset. Amplitude, latency to peak, and latency to onset were determined for each CR according to the following algorithms. Latency to onset was defined as the initial point of upward deflection in the response slope that was 2 SDs above baseline slope variation. Latency to peak was the time between the onset of the CS and the peak of the response. Amplitude was defined as the difference between the peak of each response during the $1800 \mathrm{msec}$ after CS onset and the average eyelid position for the $200 \mathrm{msec}$ baseline period. All valid trials were included in percentage and amplitude measures, while latencies to onset and to peak were determined only for CRs.

Each animal was trained for 10 daily sessions before the ipsilateral cerebellar cortex was removed by aspiration. In all cases an attempt was made to remove the anterior lobe, which is situated directly rostral and rostroventral to the cerebellar nuclei. After at least 1 week for recovery, animals were trained for 2 additional days to determine the effect of the lesion on the amplitude, percentage, and timing of CRs. Rabbits were then presented 10 daily extinction sessions composed of $109 \mathrm{CS}$ alone trials.

Tissue analysis. Rabbits were sacrificed with sodium pentobarbital $(80 \mathrm{mg} / \mathrm{kg}, \mathrm{i} . \mathrm{v}$.$) and the brains were fixed by intracardial perfusion of$ $10 \%$ formalin. Lesion extent was determined by gross inspection and recorded on schematic representations of the cerebellum reproduced from Larsell (1970). Cerebella were subsequently embedded in an albumin/gelatin mixture and sectioned at a thickness of $80 \mu \mathrm{m}$ using standard histological techniques. Examination of these slices following cresyl violet staining revealed the impact of the lesion on cerebellar nuclei and cerebellar peduncles.

Statistical analyses. To examine the effects of each lesion on conditioned response timing, separate $t$ tests for latency to onset and latency to peak were performed on the last 25 conditioned responses before the lesion and the first 25 conditioned responses following the lesion for each animal. A two-way, mixed analysis of variance (ANOVA) was performed on the percentage conditioned responding data for the last prelesion day and the 10 extinction training sessions to examine the lesion effects on extinction. A one-way, repeated-measures ANOVA for percentage conditioned responding was performed on the extinction training data for the experimental group to characterize the rate of extinction for these animals. To test for differences in prelesion training a two-way repeated measures ANOVA was performed on the percentage conditioned responding data for the 10 prelesion training sessions.
A one-way, repeated measures ANOVA for response amplitude was used to analyze the stimulus generalization data. Multiple comparisons were made using the Tukey test and two-tailed distributions with a minimum criterion of $p<0.05$ were used for all tests.

\section{Results}

Ten days of prelesion training promoted robust acquisition of conditioned eyelid responses (left panel, Fig. $1 A$ ) with no significant differences between experimental and control groups. Following surgical aspiration of the cerebellar cortex, postlesion retention of CRs was tested for $2 \mathrm{~d}$ (center panel, Fig. 1A). Animals with less than $50 \%$ retention of CRs during these $2 \mathrm{~d}$ were excluded from further study ( $n-13$; range - $046 \%$, median $=17 \%$ ). Histological analysis revealed obvious damage to the interpositus nucleus and/or superior cerebellar peduncle for ten of these animals. For the animals with greater than 50\% retention, separate $t$ tests $(p<0.001)$ for latency to peak data were used as the criterion for significant changes in response timing. Six animals met this criterion and were included in the experimental group. Five of these rabbits also displayed significant decreases in latency to onset $(p<0.001)$, while the sixth rabbit showed a less reliable decrease $(p<0.05)$. The remaining animals had no significant change in response latencies $(p>$ $0.05)$ and comprised the control group $(n=12)$. Both groups were then given 10 daily sessions of extinction training (109 CS-alone trials per session). Experimental animals showed no significant decrease in CRs over the $10 \mathrm{~d}$ of testing, whereas control animals displayed rapid extinction of CRs reaching asymptote in 2-3 d (right panel, Fig. 1A). A two-way, mixed ANOVA of the last retention day and the 10 extinction days revealed a significant lesion by session interaction effect $[F(10,161)=10.142, p<0.001]$. A one-way, repeated-measures ANOVA for the experimental group indicated no significant change over the $10 \mathrm{~d}$ of extinction $[F(9,45)=1.29, p>$ 0.05].

Subsequent gross anatomical cxamination revealed cxtensive damage to the anterior lobe for all six experimental animals (Fig. $1 B$ ), whereas control animals showed little or no anterior lobe damage. A photograph of representative lesions for an experimental (left) and control animal (right) is presented in Figure 2. In the experimental animal the left hemisphere of the cerebellar cortex, including the anterior lobe, is completely removed. In the control animal only the paramedian, HVI and portions of the ansiform lobules are removed. The behavioral data for these two rabbits (truncated at day 16) are shown below. These observations are consistent with previous data suggesting that damage to the anterior lobe is required to disrupt response timing (Perrett et al., 1993) and indicate that this region is involved in conditioned eyelid responses.

To insure that the lesions have not simply unmasked short latency, unlearned responses we examined stimulus specificity. Conditioned eyelid responses are stimulus specific in that responses decline to the degree that a probe stimulus differs from the training CS (e.g., Moore, 1972). In contrast, unlearned responses unmasked by the lesions should not by definition show specificity for the previously trained CS. On the last day prior to the lesion and on the second postlesion day, unreinforced probe trials at four frequencies were presented. Figure 3 illustrates that the postlesion eyelid responses for experimental animals are significantly larger for the training CS than for the other stimuli. For half of the animals the training CS was a $0.5 \mathrm{kHz}$ tone (left panel) and for the remaining animals the CS was a 5 

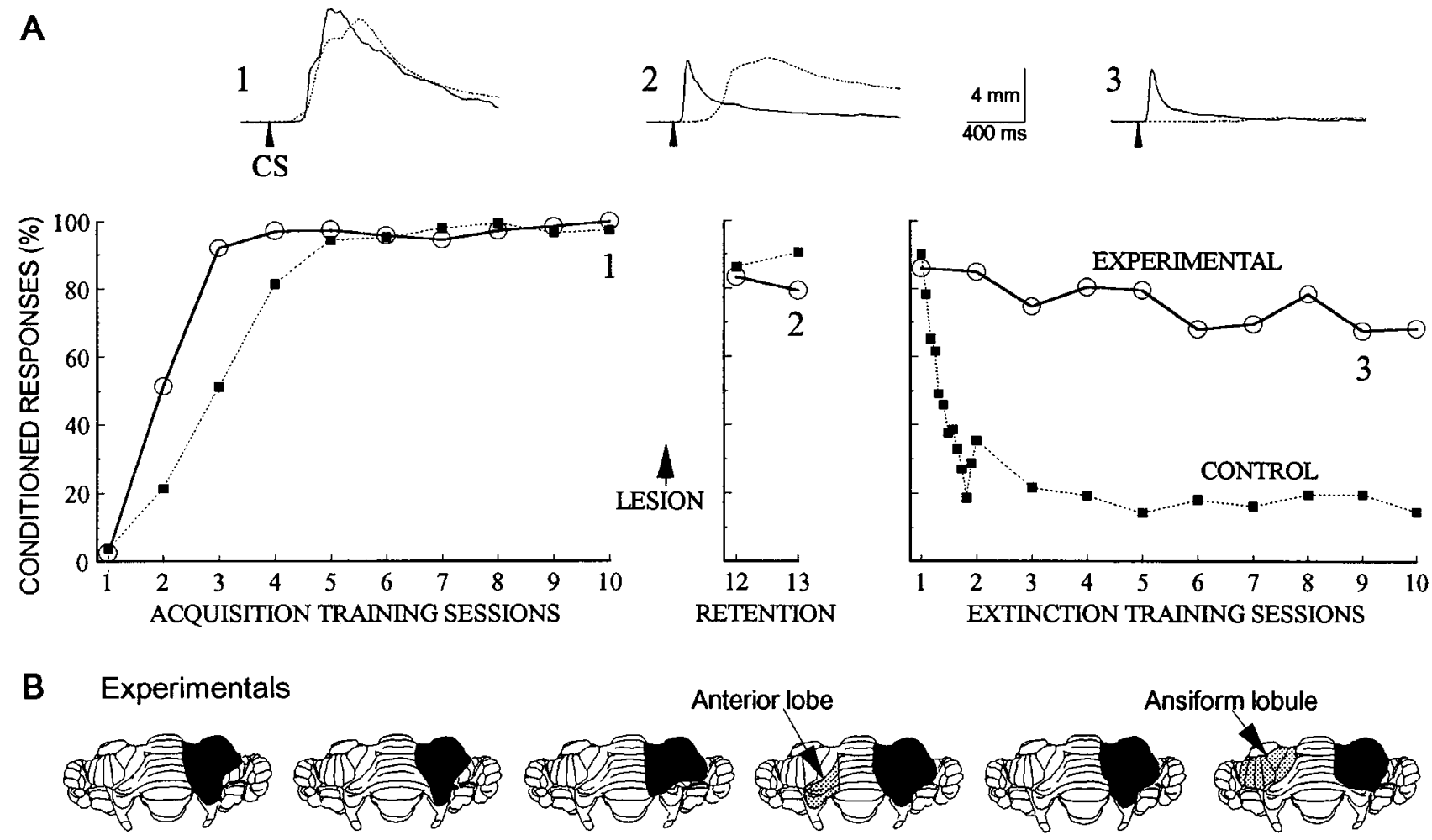

\section{Controls}
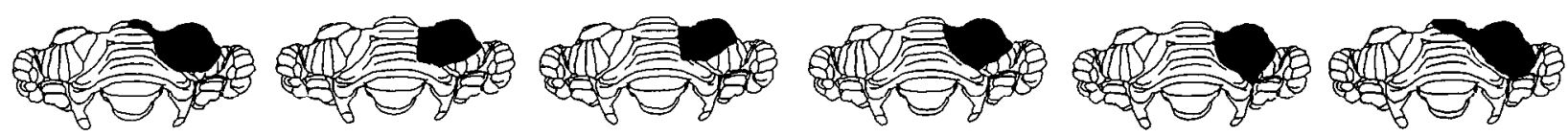

Figure 1. Lesions of the cerebellar cortex that include the anterior lobe disrupt conditioned response timing and prevent subsequent extinction. $A$, Conditioned responses (percentage) for the experimental and control groups are plotted over 22 daily training sessions. Sample eyelid responses taken from the times indicated in the graph are shown above; solid traces are from an experimental animal and dotted traces from a control. Left panel, acquisition of conditioned responses over ten days of paired CS-US trials. Aspiration lesions of the cerebellar cortex were made at the end of acquisition training. Center panel, two postlesion training sessions revealed robust retention of conditioned responses. Lesions that included damage to the anterior lobe disrupted the timing of conditioned responses (see sample responses). These animals were included in the experimental group $(n=6)$, whereas animals with normal response timing were the control group $(n=12)$. Right panel, during 10 daily sessions of CS-alone trials, control animals showed extinction that reached asymptote in 2-3 d whereas experimental animals showed no significant decrement in conditioned responding over $10 \mathrm{~d}$. The rapid rate of extinction for the control group on the first day is shown by plotting the data in 12 nine trial blocks. $B$, Reconstructions of the lesions for all 6 experimental animals and for the six largest lesions of the 12 control animals are shown schematically.

$\mathrm{kHz}$ tone (right panel). The two groups of animals $(n=4$ total; two rabbits were not tested) were analyzed statistically by combining the training stimulus (i.e., the CS) data for each animal. A one-way repeated measures ANOVA of the postlesion responses indicated a significant difference between the responses elicited by each stimulus $[F(3,9)=6.786, p<0.025]$. A subsequent Tukey test revealed that the training CS responses were significantly greater $(p<0.05)$ than both the $0.5 / 5 \mathrm{kHz}$ and the $8 \mathrm{kHz}$ responses. This stimulus specificity indicates that the postlesion eyelid responses are not unlearned responses unmasked by the lesion.

\section{Discussion}

We find that lesions of the cerebellar cortex, when they include damage to the anterior lobe, prevent extinction of conditioned eyelid responses. In contrast, extinction appears to be normal in animals with large lesions of the cerebellar cortex that spare the anterior lobe. In addition, the present data replicate previous observations (Perrett et al., 1993) that damage to the anterior lobe disrupts the learned timing of CRs. Indeed, postlesion disruption of response timing predicted subsequent failure to extinguish. Moreover, these inappropriately timed, inextinguishable responses display stimulus specificity; response magnitudes decline to the extent that a probe stimulus differs from the CS. This observation indicates that the postlesion responses are Pavlovian CRs under stimulus control rather than unlearned blinks unmasked by the lesion. These data suggest that the anterior lobe of the cerebellar cortex is necessary for both response timing and extinction of CRs.

The training protocol used in this study, in which CRs were acquired prior to the lesion, provides a behavioral assay to supplement anatomical assessment of the lesion extent. First, postlesion retention of CRs indicates that the lesion has not damaged regions necessary for the expression of responses, such as the anterior interpositus nucleus (McCormick and Thompson, 1984; Yeo et al., 1985a) or the superior cerebellar peduncle (McCor- 


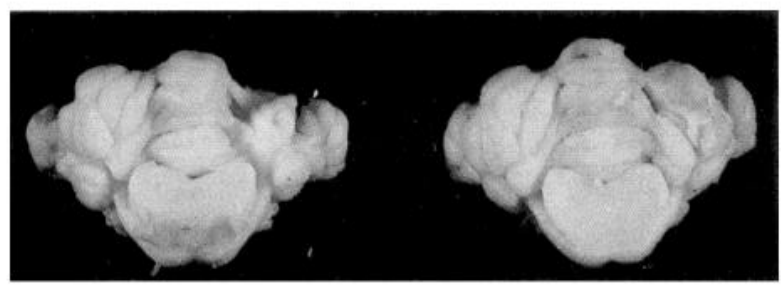

EXPERIMENTAL

CONTROL

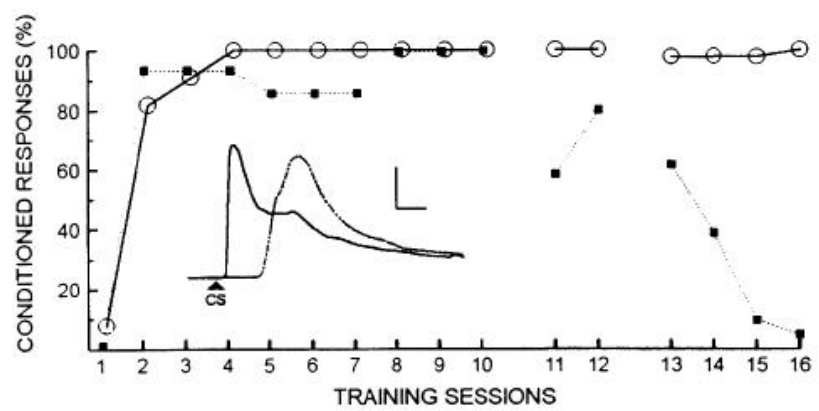

Figure 2. Behavioral data and a photograph of cerebella for representative experimental (solid lines) and control animals (dotted lines). The lesion in the experimental animal (left) involved virtually all of the left hemispheric cerebellar cortex, including the anterior lobe and the paramedian, ansiform, and HVI lobules (see labels on schematics in Fig. 1). The aspiration of the control animal (right) removed the paramedian, HVI, and portions of the ansiform lobules, but spared the anterior lobe. The inset shows average responses from the five CS-alone trials during session 12. Calibration: $2 \mathrm{~mm}, 200 \mathrm{msec}$.

mick et al., 1982). This assurance is critical given the close proximity of these regions to the cerebellar cortex and the ease with which they can be unintentionally damaged. Second, attending to the timing of the postlesion responses provides an indication of damage to regions of the cerebellar cortex that are important for eyelid conditioning. We note, however, that for all animals these functional indications of the lesion extent were confirmed with anatomical and histological analyses.

The observation that the anterior lobe is critically involved in eyelid conditioning has important implications in relating the present findings to previous studies investigating cerebellar cortex involvement in eyelid conditioning. These studies have re- ported that CRs were either abolished (Yeo et al., 1985b; Yeo and Hardiman, 1992) or, like the present data, were spared by cerebellar cortex lesions (McCormick and Thompson, 1984; Perrett et al., 1993). Studies reporting abolition of CRs have implicated the deepest portions of lobule HVI as a region of cerebellar cortex important for eyelid conditioning (Yeo et al., 1985b; Yeo and Hardiman, 1992). However, our lesions always included at least portions of lobule HVI and ansiform lobule, but only those lesions involving substantial anterior lobe damage produced significant effects on response timing and extinction. Complete lesions of lobule HVI and the ansiform lobule, sparing the anterior lobe, significantly affected neither the timing nor extinction of CRs. These data suggest that Purkinje cells involved in eyelid conditioning are either (1) distributed in both HVI and anterior lobe such that both regions must be damaged to obtain robust deficits in timing and extinction, or (2) located only in the anterior lobe.

A number of observations taken together support the hypothesis that two sites of cerebellar plasticity are responsible for the motor expression of conditioned eyelid responses. First, previous studies suggest that CSs activate mossy fiber inputs to the cerebellum (Steinmetz et al., 1987, 1989), USs activate climbing fiber inputs to the cerebellum (McCormick et al., 1985; Mauk et al., 1986), and cerebellar output via the interpositus nucleus is required for the expression of CRs (McCormick and Thompson, 1984; Yeo et al., 1985a). Second, the disruption of learned response timing produced by anterior lobe lesions, coupled with the finding that differently timed CRs can be trained using stimulation of mossy fibers in the cerebellum as the CS (Steinmetz, 1990; Perrett and Mauk, 1992), suggests that temporal discrimination and synaptic plasticity in the cerebellar cortex are involved in response timing. Third, the retention of improperly timed CRs following anterior lobe lesions is consistent with two sites of synaptic plasticity, with one site of plasticity not in the cerebellar cortex. The interpositus nucleus is implicated as this site by the following observations: (1) animals can be trained with mossy fiber stimulation as the CS, (2) animals retain CRs following lesions of the cerebellar cortex, and (3) stimulation of the interpositus nucleus in naive animals elicits robust eyeblinks (McCormick and Thompson, 1984; Chapman et al., 1988).

Based on the above observations we propose the following
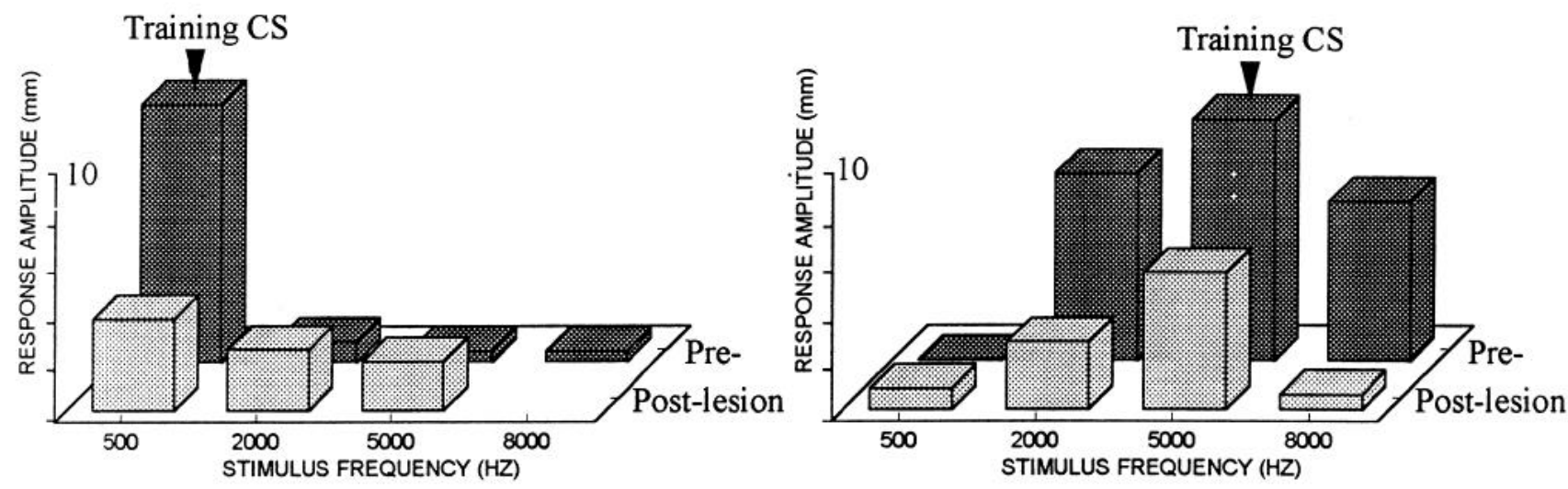

Figure 3. Experimental animals display postlesion eyelid responses that are stimulus specific. Four different probe stimuli were presented to animals on the last day prior to the lesions and again on the second session after the lesion. For half of the animals the training CS was a $0.5 \mathrm{kHz}$ tone (left panel) and for the remaining animals the CS was a $5 \mathrm{kHz}$ tone (right panel). In each case the post lesion responses were larger for the training CS than for the other three probe stimuli. This specificity to the training CS indicates that the postlesion responses are Pavlovian responses under stimulus control. 


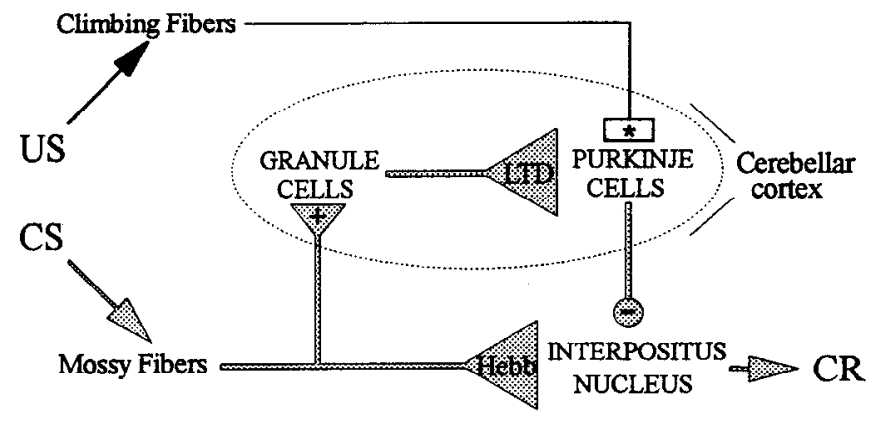

Figure 4. Schematic representation of the pathways relevant to the proposed model of cerebellar involvement in Pavlovian conditioning. Cerebellar pathways activated by the CS and involved in the expression of the conditioned response are shown in gray. Pathways activated by the US and that are responsible for its reinforcing properties are shown with solid lines. The large triangles represent the assumed sites of synaptic plasticity and the rules for induction; granule Purkinje synapses display generalized long-term depression (LTD) and mossy fiber synapses in the interpositus nucleus display generalized Hebbian plasticity (Hebb). These assumptions predict that the cerebellar cortex is required for the extinction of conditioned eyelid responses.

hypothesis to account for the disruption of extinction produced by anterior lobe lesions (Fig. 4). First, granule cell synapses onto Purkinje cells $(\mathrm{Gr} \rightarrow \mathrm{Pkj})$ in the cerebellar cortex display generalized long-term depression (LTD) as supported by a number of in vivo and in vitro studies (Sakurai, 1987; Ito, 1989; Linden et al., 1991) - that is, $\mathrm{Gr} \rightarrow$ Pkj synapses decrease in strength when active in the presence of a climbing fiber input and increase in strength when active without a climbing fiber input. Second, mossy fiber synapses onto interpositus nucleus cells (MF $\rightarrow$ nuc) display a form of generalized Hebbian plasticity (e.g., Hebb, 1949; Brown et al., 1990; Dudek and Bear, 1993). We assume that $\mathrm{MF} \rightarrow$ nuc synapses increase in strength when active during transient decreases in Purkinje cell inhibitory input and decrease in strength when active during robust Purkinje cell input. This hypothesis suggests that input from the cerebellar cortex controls the induction of plasticity at the $\mathrm{MF} \rightarrow$ nuc synapses.

These assumptions predict that paired presentations of CS and US promote acquisition of CRs by inducing LTD at specific, $\mathrm{CS}$-activated $\mathrm{Gr} \rightarrow \mathrm{Pkj}$ synapses. The resulting learned decrease in Purkinje activity during the CS would have the dual effect of directly promoting CRs and permitting Hebbian increases in CSactivated $\mathrm{MF} \rightarrow$ nuc synapses. Therefore, CRs in a well-trained animal would result from increased synaptic activation of interpositus cells and decreased inhibition of the same cells via LTD in the cerebellar cortex.

Because we assume these forms of plasticity are generalized, these synaptic changes would be reversed during extinction. CSalone presentations would potentiate the $\mathrm{Gr} \rightarrow \mathrm{Pkj}$ synapses, restoring Purkinje inhibition during the CS. This would directly reduce $\mathrm{CRs}$ and promote Hebbian decreases in $\mathrm{MF} \rightarrow$ nuc synapses. In the context of this hypothesis, removing the cerebellar cortex should abolish the ability of $\mathrm{MF} \rightarrow$ nuc synapses to undergo Hebbian changes. Without inhibition from Purkinje cells $\mathrm{MF} \rightarrow$ nuc synapses could not undergo Hebbian decreases, which would prevent extinction.

Intracellular recordings from cerebellar nucleus cells (Llinás and Mühlethaler, 1988) suggest an example of how transient release from inhibition might be important for Hebbian increases at the $\mathrm{MF} \rightarrow$ nuc synapses. Nucleus cells displayed a voltagesensitive calcium conductance that was revealed following re- lease from hyperpolarizing pulses, but not by depolarization from the cell's resting potential. An interesting speculation, for which there is no supporting evidence, is that elevated intracellular calcium levels could trigger increases in coactive $\mathrm{MF} \rightarrow$ nuc synapses. This hypothesis leads to the prediction that lesions of the anterior lobe should also prevent acquisition of CRs, which is currently being tested.

This dual site plasticity hypothesis is consistent with our previous suggestion that variations in the subsets of granule cells activated throughout the CS (Perrett et al., 1993) mediate response timing. Recent large scale computer simulations of mossy fibers, granule cells, and Golgi cells support this suggestion (Buonomano and Mauk, 1994). With such a time-varying CS representation, the associative nature of LTD would ensure that only those subsets of $\mathrm{Gr} \rightarrow$ Pkj synapses active near US onset would decrease in strength. During a CS presented to a trained animal, Purkinje cell inhibition of nucleus cells would be maintained until just before the US, mediating a properly timed CR. Following removal of the cerebellar cortex, the absence of Purkinje cell inhibition would permit previously strengthened mossy fiber synapses to elicit action potentials in the interpositus neurons without delay, giving rise to CRs with very short, fixed latencies.

Our assumptions regarding two sites of plasticity suggest a possible explanation for the apparent contradiction in studies examining retention of CRs following lesions of the cerebellar cortex. With plasticity in the cortex controlling plasticity in the interpositus nucleus as we propose, CRs could be present before the induction of significant plasticity in the nucleus. Indeed, Hebbian-like plasticity in the nucleus could continue to increase even after robust CRs are present. Thus, the degree of plasticity in the nucleus and whether cerebellar cortex lesions spare CRs might depend on the amount and type of prelesion training.

Our hypothesis also suggests an explanation to reconcile seemingly contradictory data in which reversible inactivation of the interpositus nucleus was used to examine cerebellar involvement in Pavlovian conditioning. In one study (Krupa et al., 1993) infusion of the GABA agonist muscimol during training blocked expression and acquisition of CRs. In contrast, infusion of the local anesthetic lidocaine blocked expression but not acquisition of CRs (Welsh and Harvey, 1991). Differences in the mode of action of these compounds may explain these results. Neither compound should prevent the induction of LTD in the cortex. Since lidocaine blocks both pre- and postsynaptic action potentials, it should block expression of CRs and prevent Hebbian-like plasticity in the nucleus. With lidocaine removed, the LTD in the cortex could support immediate expression of CRs. Conversely, muscimol presumably blocks expression by hyperpolarizing nucleus cells, but under our assumptions this would also promote Hebbian decreases in the strength of the $\mathrm{MF} \rightarrow$ nuc synapses activated by the CS. With muscimol removed, the decrements in $\mathrm{MF} \rightarrow$ nuc synaptic strength might counteract the LTD induced in the cortex and delay the appearance of CRs. Thus, our assumptions are consistent with the rapid expression of CRs seen following training during lidocaine infusion and the slower appearance of CRs following training during muscimol infusion.

A number of studies have demonstrated that timing and extinction of CRs can also be affected by lesions of the hippocampus. Hippocampal lesions have been reported to decrease CR latencies (Orr and Berger, 1985; Port et al., 1985, 1986; Akase et al., 1989; Moyer et al., 1990; Christiansen and Schmajuk, 1992), increase response latencies (Port et al., 1986) or have no 
effect on timing (Orr and Berger, 1985; Port et al., 1985; Moyer et al., 1990). Likewise, studies have demonstrated that hippocampal lesions retard rates of extinction (Orr and Berger, 1985; Akase et al., 1989; Moyer et al., 1990), enhance rates of extinction (Akase et al., 1989), or have no effect on extinction (Schmaltz and Theios, 1972; Berger and Orr, 1983). It is difficult to identify the factors that lead to these variable results since these studies differed along a number of potentially important dimensions, including the type of training (i.e., delay vs trace procedures) and the interstimulus or trace intervals used.

These data suggest the possibility that the hippocampus and the cerebellum interact to influence response timing and extinction. Understanding the nature of this putative interaction may be aided by the resolution of a number of issues. First, it seems important to identify reasons for the variable lesion results. Drawing parallels from the present data, one possibility is that there is(are) critical region(s) of hippocampus important for eyelid conditioning and that the variable behavioral results arise from lab to lab variations in the size or location of the lesions. Another possibility suggested by the data is that hippocampal lesions have greater effects on responses promoted by more complex or difficult training protocols. Second, to identify precisely the role of the hippocampus in eyelid conditioning it is also important to examine the cffects of hippocampal lesions made after acquisition of CRs (Schmaltz and Theios 1972; Port et al., 1986). This avoids the potential complication that acquisition with a damaged hippocampus utilizes neural pathways or mechanisms that are different than normal (see Mauk and Thompson, 1987).

Two studies that examined the effects of cerebellar interpositus nucleus lesions on conditioning-related hippocampal unit activity may provide some insight into the precise nature of cerebellar/hippocampal intcractions. Hippocampal pyramidal cells show increased responding during the CS that parallels both the development of CRs over trials and the timing of CRs (i.e., Berger et al., 1976; Berger and Thompson, 1978). These observations have prompted the suggestion that hippocampal activity forms a temporal model of the CR which may play an important role in response timing. Clark et al. (1984) demonstrated that interpositus lesions made after initial acquisition abolish both the behavioral and hippocampal responses. Training the other eye subscquently promoted the usual conditioning-related hippocampal activity. Sears and Steinmetz (1990) demonstrated that interpositus lesions prevent the development of both CRs and conditioning-related hippocampal activity. Again, conditioningrelated hippocampal activity was seen with subsequent training of the other eye. These data suggest that interactions between these structures may involve information flow from the cerebellum to the hippocampus and perhaps not from hippocampus to cerebellum. Observations of this nature should contribute to a better understanding of possible cerebellar/hippocampal interactions.

In summary, cerebellar cortex lesions which involve the anterior lobe produce robust deficits in the learned timing and rate of extinction of conditioned eyelid responses. These data are consistent with a model for eyelid conditioning in which we state explicit rules for synaptic plasticity at two sites in the cerebellum. This model predicts that the cerebellar cortex is necessary for adapting movements influenced by the cerebellum. In this vicw, which provides accounts for existing data and may resolve certain controversies, lesions of the cerebellar cortex may not always abolish CRs but, as we have shown, disrupt the ability to extinguish previously learned movements.

\section{References}

Akase E, Alkon DL, Disterhoft JF (1989) Hippocampal lesions impair memory of short-delay conditioned eye blink in rabbits. Behav Neurosci 103:935-943.

Berger TW, Orr WB (1983) Hippocampectomy selectively disrupts discrimination reversal conditioning of the rahbit nictitating membrane response. Behav Brain Res 8:49-68.

Berger TW, Thompson RF (1978) Neuronal plasticity in the limbic system during classical conditioning of the rabbit nictitating mem brane response. I. The hippocampus. Brain Res 145:323-346.

Berger TW, Alger B, Thompson RF (1976) Neuronal substrate of classical conditioning in the hippocannus. Science 192:483-485.

Brown TH, Kairiss EW, Keenan CL (1990) Hebbian synapses: biophysical mechanisms and algorithms. Annu Rev Neurosci 13:475511.

Buonomano DV, Mauk MD (1994) Neural network model of the cerebellum: temporal discrimination and the timing of motor responses. Neural Comp 6:38-55.

Chapman PF, Steinmetz JE, Thompson RF (1988) Classical conditioning does not occur when direct stimulation of the red nucleus or cerebellar nuclei is the unconditioned stimulus. Brain Res 442:97104.

Christiansen BA, Schmajuk NA (1992) Hippocampectomy disrupts the topography of the rat eyeblink response during acquisition and extinction of classical conditioning. Brain Res 595:206-214.

Clark GA, McCormick DA, Lavond DG, Thompson RF (1984) Effects of lesions of cerebellar nuclei on conditioned behavioral and hippocampal neuronal responses. Brain Res 291:125-136.

Dudek SM, Bear MF (1993) Bidirectional long-term modification of synaptic effectiveness in the adult and immature hippocampus. J Neurosci 13:2910-2918.

Hebb DO (1949) The organization of behavior. New York: Wiley.

Ito M (1989) Long term depression. Annu Rev Neurosci 12:85-102.

Krupa DJ, Thompson JK, Thompson RF (1993) Localization of a memory trace in the mammalian cerebellum. Science 260:989-991.

Larsell O (1970) The comparative anatomy and histology of the cerebellum from monotremes through apes. Minneapolis: University of Minnesota.

Linden DJ, Dickinson MH, Smeyne M, Connor JA (1991) A long-term depression of AMPA currents in cultured cerebellar Purkinje neurons. Neuron 7:81-89.

Llinás R, Mühlethaler M (1988) Electrophysiology of guinea-pig cerebellar nuclear cells in the in vitro brain stem-cerebellar preparation. J Physiol (Lond) 404:241-258.

Mauk MD, Ruiz BP (1992) Learning-dependent timing of Pavlovian eyelid responses: differential conditioning using multiple interstimulus intervals. Behav Neurosci 106:1-16.

Mauk MD, Thompson RF (1987) Retention of classically conditioned eyelid responses following acute decerebration. Brain Res 403:8995.

Mauk MD, Steinmetz JE, Thompson RF (1986) Classical conditioning using stimulation of the inferior olive as the unconditioned stimulus. Proc Natl Acad Sci USA 83:5349-5353.

McCormick DA, Thompson RF (1984) Cerebellum: essential involvement in the classically conditioned eyelid response. Science 223:296299.

McCormick DA, Guyer PE, Thompson RF (1982) Superior cerebellar peduncle lesions selectively abolish the ipsilateral classically conditioned nictitating membrane/eyelid responses of the rabbit. Brain Res 244:347-350.

McCormick DA, Steinmetz JE, Thompson RF (1985) Lesions of the inferior olivary complex cause extinction of the classically conditioned nictitating membrane/eyelid response. Brain Res 359:120-130.

Moore JW (1972) Stimulus control: studies of auditory generalization in rabbits. In: Classical conditioning. II. Current research and theory (Black AH, Prokasy WF, eds), pp 206-230. New York: Appleton.

Moyer JR Jr, Deyo RA, Disterhoft JF (1990) Hippocampectomy disrupts trace eye-blink conditioning in rabbits. Behav Neurosci 104: 243-252.

Orr WB, Berger TW (1985) Hippocampectomy disrupts the topography of conditioned nictitating membrane responses during reversal learning. Behav Neurosci 99:35-45. 
Perrett SP, Mauk MD (1992) Temporal discrimination and Pavlovian eyelid responses: cerebellar versus pre-cerebellar mechanisms. Soc Neurosci Abstr 18:1560.

Perrett SP, Ruiz BP, Mauk MD (1993) Cerebellar cortex lesions disrupt learning-dependent timing of conditioned eyelid responses. $\mathrm{J}$ Neurosci 13:1708-1718.

Port RL, Mikhail AA, Patterson MM (1985) Differential effects of hippocampectomy on classically conditioned rabbit nictitating membrane response related to interstimulus interval. Behav Neurosci 99 $200-208$.

Port RL, Romano AG, Steinmetz JE, Mikhail AA, Patterson MM (1986) Retention and acquisition of classical trace conditioned responses by rabbits with hippocampal lesions. Behav Neurosci 100 : $745-752$.

Sakurai M (1987) Synaptic modification of parallel fibre-Purkinje cell transmission in in vitro guinea-pig cerebellar slices. J Physiol (Lond) 394:463-480.

Schmaltz LW, Theios J (1972) Acquisition and extinction of a classically conditioned response in hippocampectomized rabbits (Oryctolagus cuniculus). J Comp Physiol Psychol 79:328-333.

Sears LL, Steinmetz JE (1990) Acquisition of classically conditionedrelated activity in the hippocampus is affected by lesions of the cerebellar interpositus nucleus. Behav Neurosci 104:681-692.

Steinmetz JE (1990) Classical nictitating membrane conditioning in rabbits with varying interstimulus intervals and direct activation of cerebellar mossy fibers as the CS. Behav Brain Res 38:97-108.

Steinmetz JE, Logan CG, Rosen DJ, Thompson JK, Lavond DG, Thompson RF (1987) Initial localization of the acoustic conditioned stimulus projection system to the cerebellum essential for classical eyelid conditioning. Proc Natl Acad Sci USA 84:3531-3535.

Steinmetz JE, Lavond DG, Thompson RF (1989) Classical conditioning in rabbits using pontine nucleus stimulation as a conditioned stimulus and inferior olive stimulation as an unconditioned stimulus. Synapse $3: 225-233$

Thompson RF (1986) The neurobiology of learning and memory. Science 233:941-947.

Welsh JP, Harvey JA (1991) Pavlovian conditioning in the rabbit during inactivation of the interpositus nucleus. J Physiol (Lond) 444: $459-480$.

Yeo CH (1991) Cerebellum and classical conditioning of motor responses. Ann NY Acad Sci 627:292-303.

Yeo CH, Hardiman MJ (1992) Cerebellar cortex and eyeblink conditioning: a reexamination. Exp Brain Res 88:623-638.

Yeo CH, Hardiman MJ, Glickstein M (1985a) Classical conditioning of the nictitating membrane response of the rabbit $I$. lesions of the cerebellar nuclei. Exp Brain Res 60:87-98

Yeo CH, Hardiman MJ, Glickstein M (1985b) Classical conditioning of the nictitating membrane response of the rabbit II. Lesions of the cerebellar cortex. Exp Brain Res 60:99-113. 